\title{
Automated Bearing Fault Diagnosis using Packet Features of Vibration Signal and Gaussian Support Vector Machine
}

\author{
Rajeev Kumar Chauhan, Dipti Saxena, Jai Prakash Pandey
}

\begin{abstract}
Effective detection of the bearing fault and, specifically performance dilapidation assessment of a bearing is the topic of intensive analysis that may scale back prices and therefore the nonscheduled down time. This article presents an adaptive approach that is based on Bhattacharya space ranking method and dimensional reduction method as general discriminate analysis (GDA) with Gaussian support vector machine (GSVM) to accurately detect the defects of rolling bearing. For this investigation, first, vibration signal generated by rolling bearing was disintegrated to five levels employing wavelet packet (WP) method. Sixty three logarithmic wavelet packet features (LWPFs) were taken out from five level disintegrated vibration signals. After this, sixty three features were ranked by Bhattacharya space and top ten LWPFs were chosen. The top ten features were reduced to a new feature using GDA for effective detection and then applied to GSVM for detection of bearing fault. The experimental results show that new automated diagnosing approach attained classifier performance parameters as sensitivity (SE) or true positive rate, specificity $(S P)$ or true negative rate, accuracy $(A C)$ and positive prediction value $(P P V)$ of 100, 98.50, 100 and $99.67 \%$ for inner raceway (IR) and, $A C$ : 99.49, SE: 100, SP: 98.78 and PPV: 99.87 for ball bearing $(B B)$ at $0.18 \mathrm{~mm}$ diameter faults.
\end{abstract}

Keywords: Bhattacharya space ranking method, ball bearing (BB) defect, Gaussian support vector machine, General Discriminate Analysis, inner race (IR), wavelet packet.

\section{INTRODUCTION}

The most critical but vulnerable mechanical components in a rotating machine are rolling element bearings. A bearing failure can result in a complete machine breakdown resulting in unintended production process interruption and financial loss. According to IEEE induction motor reliability study [1], bearing faults are the foremost frequent faults in induction machines around $42 \%$, stator faults nearby $37 \%$ and rotor failures approximately $10 \%$. Therefore, failure to detect

rolling bearing elements is of prime importance and should be supervised on a priority basis to maximize the utilization

of the induction motor's operating life. For this, efficient detection technique and features (attributes) taken from

Revised Manuscript Received on March 5, 2020.

Rajeev Kumar Chauhan is with Electrical \& Electronics Engineering Department, IMS Engineering College, Ghaziabad-201009 (UP) India (Contact No. (+91)9873479813; e-mail: mmmec.rkc@ gmail.com).

Dipti Saxena is with Electrical Engineering Department, MNIT, Jaipur302017 (Rajasthan) India. (e-mail: diptisx @gmail.com).

Jai Prakash Pandey is with Electrical Engineering Department, KNIT, Sultanpur-228001 (UP) India. (e-mail: tojppandey @rediffmail.com). vibration signal of rolling bearing used in motor are needed. Commonly, there are two types of bearings; rolling element and fluid bearing. The rolling element bearings are usually used for complex moment loads with lesser friction and in small and moderate dimension machines while the fluid bearing can handle very large loads with low friction and used in large dimension machines [2]. Nowadays, rolling element bearing fault analysis has been applied extensively. There are old methods like; temperature observance, oil analysis and stator current methods, which can be used to detect bearing defects [3], [4]. But due to high cost and noise impact, these detection techniques are not acceptable. Bearing failure analysis using vibration signal has become one of the leading parameters for researchers in recent years [5], [6]. The nonlinear vibration signals generated from bearings is generally affected by random signal as; noise and signal modulation [7]. For this, Xu et al. [8] suggested a Fuzzy Model Controller (FMC) with neural network classifier to analyze the degree of bearing faults performance degradation using features from vibration signal. Yu et al. [9] suggested a simple and efficient machine fault diagnostic algorithm supported on Gaussian Mixture Model (GMM), the simulated results presented that GMM could reliably detect not solitary type of bearing fault and the degree of degradation in machine performance. Pan et al. [10] employed a hybrid model as support vector and Fuzzy c-means for bearing fault dilapidation. In a bulky dimension rotating machine, Sun et al. [11] obtained a fault diagnosing by evolving kernel principal component analysis, which transmutes the features of vibration signal to a more effective nonlinear features and improve the detection performance of a bearing fault diagnosis. Widodo et al. [12] extracted nonlinear features from vibration signal and support vector machine (SVM) to classify faults of bearing used in induction motor.

From the above cited references we can notice that there are two vital aspects of faults assessment and detection accurately and automatically: feature extraction methods from vibration signal and efficient classifier. Ancient diagnosing techniques extract attribute (features) from vibration signals in time, frequency and time-frequency (T-F) domain. Though, due to the chaos operating state of rotating bearings as high speed, friction, over loads and alternative nonlinear factors, it's terribly troublesome to classify the faulty and healthy bearing and particularly the degree of performance degradation of the rotator bearing using time or frequency domain extraction features form vibration signal.

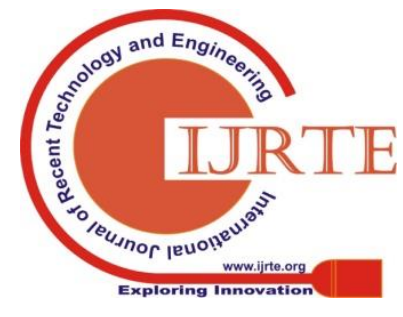


Various T-F domain methods as short time Fourier transform, empirical mode decomposition, continuous Morlet wavelet transform (CMWT), Wigner-Ville distribution (WVD), and Pseudo Wigner-Ville distribution (PWVD) plays significant role [13], [14] in detection and assessment of faulty bearings. CMWT is broadly used in T$\mathrm{F}$ domain due to the simple and independence of mother wavelet function selection [15], [16]. Still, this method cannot effectively decompose into high and low frequency band of vibration signal, which carry rich information of fault signal. Wavelet packet (WP) decomposition is one of the T-F methods that have the capability to overcome the drawbacks of wavelet transform method.

In this article, an automated algorithm, which is a combination of Bhattacharya space ranking method and dimensional reduction method as general discriminate analysis (GDA) with Gaussian support vector machine (GSVM) is presented to enhance the detection accuracy of fault diagnosis of the rolling bearing element. For this, first, the vibration signal was decomposed to five levels by Wavelet Packet (WP) method. Sixty three non-linear Logarithmic Wavelet Packet Features (LWPFs) were extracted from five level decomposed vibration signal. The flow chart of proposed algorithm is shown in Fig.1. As, the performance metrics of detection methods depends on the assortment of suitable features [17], [18]. Intended for, The Bhattacharya space method [19] was applied to choose top most ten features out of sixty three features extracted from decomposed vibration signal. Further these topmost features were transformed to a new feature by features reduction scheme commonly known as general discriminant analysis (GDA) and kernel principle component analysis (KPCA) [17], [20], [21]. The new feature was employed to train and validate the Gaussian support vector machine (GSVM) to accurately detect the bearing faults. The performance in terms of accuracy (AC), specificity (SP), sensitivity (SE) and positive

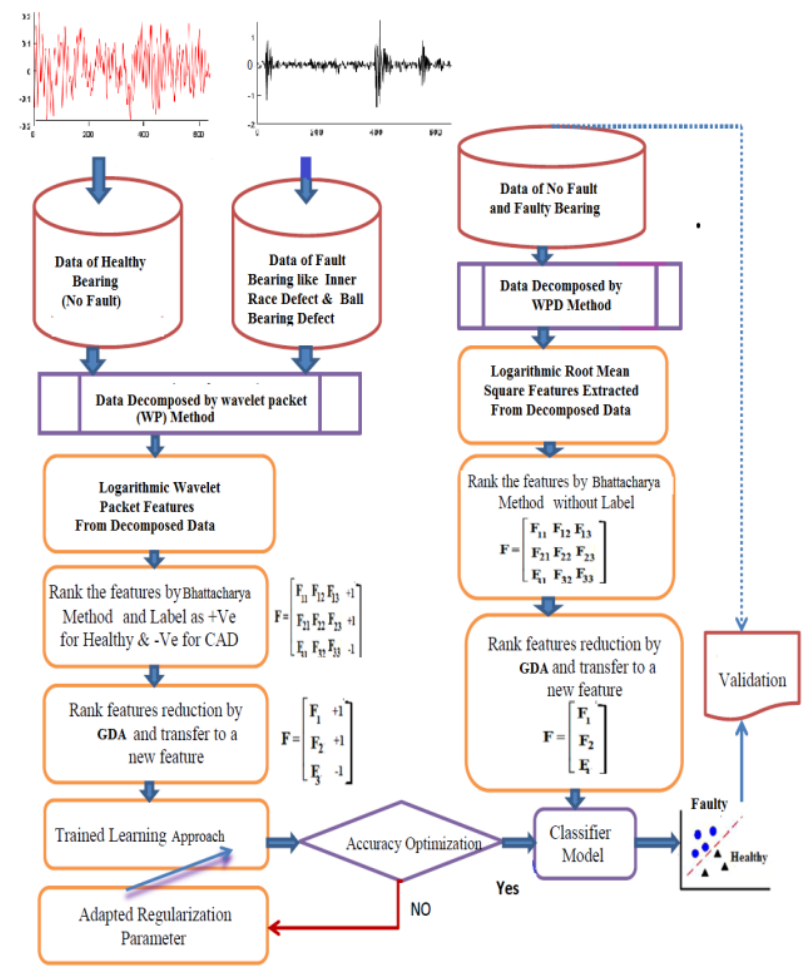

Fig. 1. Flowchart of the proposed work to detect faulty and healthy condition of bearing prediction value (PPV) of the proposed scheme was evaluated using confusion matrix.

\section{METHODOLOGY}

\section{A. Database}

The vibration standarad datasets employed in this study was taken from online obtainable bearing knowledge center website of Case Western Reserve University (CWRU) [22]. The investigational setup design by Reliance consisted of an induction motor, torsion transducer/encoder for measurement of torque, an accelerometer for collection of vibration signal, a dynamometer and electronics control circuit at loads $0 \mathrm{HP}, 1 \mathrm{HP}, 2 \mathrm{HP}$ and $3 \mathrm{HP}$. The sixteen channels were employed to collect the vibration data and were pre-proocessed in a MATLAB tool.The signals were sampled at $12 \mathrm{kHz}$ frequency. The single point faults in inner raceway (IR) and in ball bearing (BB) were introduced to the model SKF 6205-2RS JEM using electro-discharge machining (EDM) with fault diameters of 7, 14, 21, 28 mils. For this analysis, 36 database of each healthy bearing or no fault (NF), IR and $\mathrm{BB}$ bearing defects were taken at different loads for only 10 seconds. The sets of vibration signals are repersented in Table I.

Table I

Represents Symbols Of Single Point Faults In Inner Raceway (Ir) And In Ball Bearing (Bb)

\begin{tabular}{|l|c|c|c|c|}
\hline Fault Level: mils(mm) & $\mathbf{7 ( 0 . 1 8 )}$ & $\mathbf{1 4 ( 0 . 3 5 )}$ & $\mathbf{2 1}(\mathbf{0 . 5 3})$ & $\mathbf{2 8}(\mathbf{0 . 7 2})$ \\
\hline Inner Raceway & IR7 & IR14 & IR21 & IR28 \\
\hline Ball Bearing & BB7 & BB14 & BB21 & BB28 \\
\hline Healthy Bearing & \multicolumn{5}{|c|}{ No Fault (NF) } \\
\hline
\end{tabular}

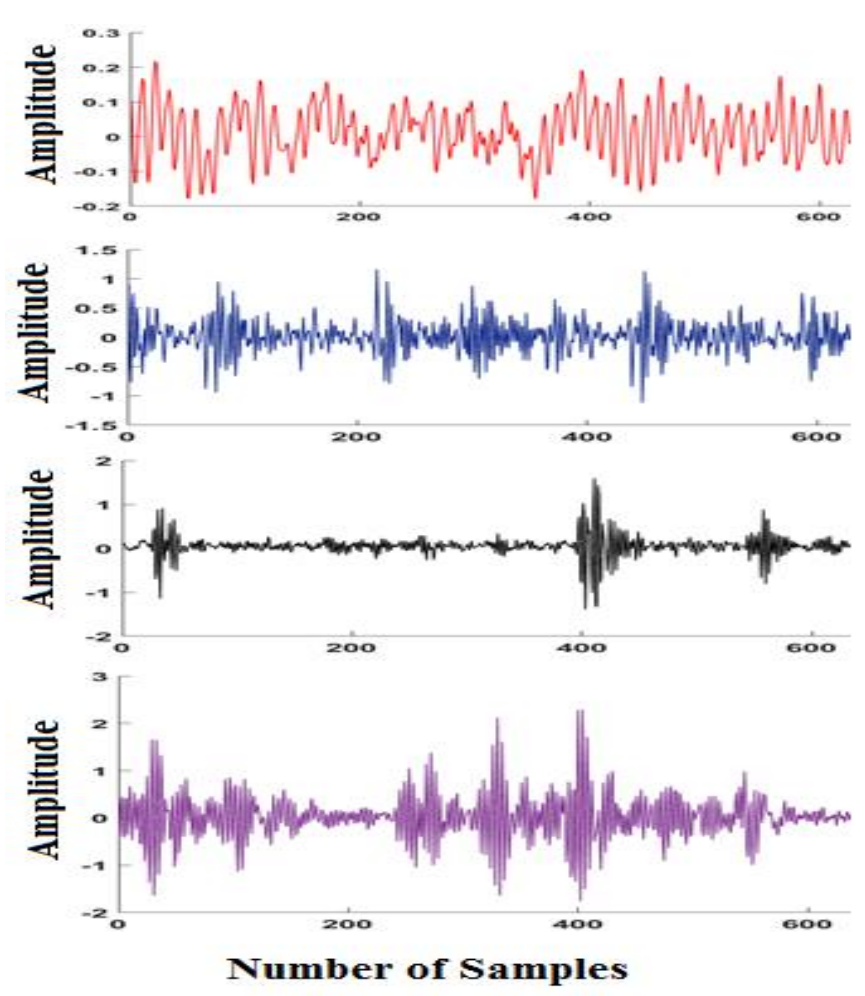

Fig. 2. Wave form of a raw data of vibration signal for (a) NF (b) IR7 (c) IR14 (d) IR21 at 0HP load. 


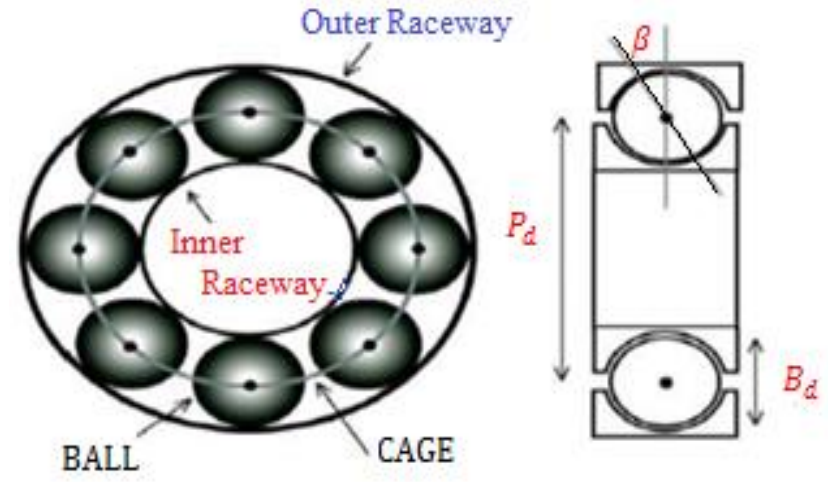

Fig 3. Front view with geometric construction of rolling element bearing

The amplitude vs number of samples of NF, IR7, IR14, IR21 and IR28 of vibration signal at 0HP load are shown in Fig. 2.

\section{B. Characteristics frequencies releted to rolling element bearing}

Bearings play an vital role to keep the rotor and stator at equidistance. Rolling element bearing is most commonly used bearing in an induction motor and consists of four essential parts: cage (separator), inner raceway, outer raceway and rolling element (roller or ball). Lubricant contamination, lubricant loss or excess lubrication, brinelling, excess loading, overheating and corrosive environments are some basic cause to bearing failure or create the defect in bearing [2]. This faults can be classified into two parts as distributed and single point defects. With all type of bearing defect, a characteristic frequency can be associated. But, distributed defect distresses the entire area of bearing elements and difficult to distinguish by discrete frequencies.

Whereas single-point defect is associated with a small region of bearing elements that create a harmonic series with fundamental frequency. The characteristic frequencies as cage defect frequency $\left(C D_{f}\right)$, outer race defect frequency $\left(O R D_{f}\right)$, inner race defect frequency $\left(I R D_{f}\right)$, and ball defect frequencies $\left(B D_{f}\right)$ are functions of the bearing geometry and rotor frequency [2]. The front view with geometric structure of rolling element ball bearing is depicted in Fig.3. Assume that $R_{f}$ is rotational frequency, $B_{d}$ is diameter of ball diameter, $P_{d}$ is pitch diameter, $B_{n}$ is total no of balls, and $\beta$ is contact angle, the equation of fault frequencies are defined as

$$
\begin{aligned}
& C D_{f}=\frac{R_{f}}{2}\left[1-\frac{B_{d}}{P_{d}} \cos (\beta)\right] \\
& I R D_{f}=\frac{B_{n}}{2}\left[1+\frac{B_{d}}{P_{d}} \cos (\beta)\right] R_{f} \\
& O R D_{f}=\frac{B_{n}}{2}\left[1-\frac{B_{d}}{P_{d}} \cos (\beta)\right] R_{f} \\
& B D_{f}=\frac{B_{n}}{2}\left[1-\frac{B_{d}^{2}}{p_{d}^{2}} \cos (\beta)^{2}\right] R_{f}
\end{aligned}
$$

These characteristic frequencies were employed in decomposition of vibration signal. The standard geometrical parameters of model SKF 6205-2RS JEM bearing were $B_{n}=9, B_{d}=318.6$ mils, $P_{d}=1537$ mils for simulation of results.

\section{Feature extraction by wavelet packet}

The wavelet packet (WP) is the extension of wavelet transform method that decompose a vibration signal into many independent high and low frequency signals referred as packets. This allows the assortment of the decomposition levels that make available for best packet feature extraction; this is advantageous for monitoring condition and diagnosis of machine failure or bearing fault [23], [24]. WP consists of a group of linearly combined wavelet functions that are generated by the algorithmic relationship given by

$$
\begin{aligned}
& W^{2 p}(x)=\sqrt{2} \Sigma_{q} S(q) W^{p}(2 x-q) \\
& w^{2 p+1}(x)=\sqrt{2} \sum_{q} g(q) w^{p}(2 x-q)
\end{aligned}
$$

Where $S(q)$ is sample of variation signal at $q=1,2, \ldots \ldots \ldots \ldots n$ and first two WP functions $W^{0}(x)=\emptyset(x)$ and $W^{1}(x)=\emptyset(x)$ are known as scaled function and wavelet coefficients (filter bank). The symbol $S(q)$ and $g(q)$ are connected to each other by $g(q)=(-1)^{q} \times S(1-q)$ and is coefficient of a pair of Quadrature Mirror Filters linked with the scaled and wavelet function [25]. These coefficients consecutively decompose the vibration signal into approximation (low frequency) and detail (high frequency) signals by the scaled and wavelet coefficients, respectively. The input vibration signal $Y(t)$ can be decomposed recursively as

$$
\begin{aligned}
& Y_{i+2,2 p}(x)=\sum_{m} S(m-2 q) Y_{i, p}(x) \\
& Y_{i+1,2 p+1}(x)=\Sigma_{m} g(m-2 q) Y_{i, p}(x)
\end{aligned}
$$

Where $Y_{\text {ipp }}(x)$ represents the wavelet coefficients at the $i^{i^{\text {th }}}$ level, $k^{\text {th }}$ sub frequency band. Hence the vibration signal $Y(t)$ can be written as

$$
Y(t)=\sum_{p=0}^{2^{i}-1} Y_{i, p}(x)
$$

Three level decomposition of vibration signal $Y(t)$ by WP is illustrated in Fig.4. In this figure, dotted mark indicates Detail (high frequency) and bold mark represents Approximation (low frequency) components. The Logarithmic Wavelet Packet Features (LWPFs) of decomposed vibration signal is defined as

$$
L W P F s=\sqrt{\frac{\left(x_{1}^{2}+x_{2}^{2}+x_{3}^{2}+----+x_{N}^{2}\right)}{N}}
$$

Where ${ }^{x_{1}}$ and $x_{2}$ etc. are samples of decomposed vibration signal and $N$ is number of samples in decomposed signal. WP generates $2^{\text {Level }}$ features at each level of decomposition of vibration signal. Therefore, the total no of features are sixty three for 5 levels decomposition of original signal.

\section{Feature subset selection and dimension reduction}

Feature subset selection is the method wherever we automatically or manually choose those features that contribute most to prediction variable or output during which we are inquisitive about [17]. It identifies the relevant features from a huge dimension features and removes the irrelevant or less important features which do not contribute much to our target 


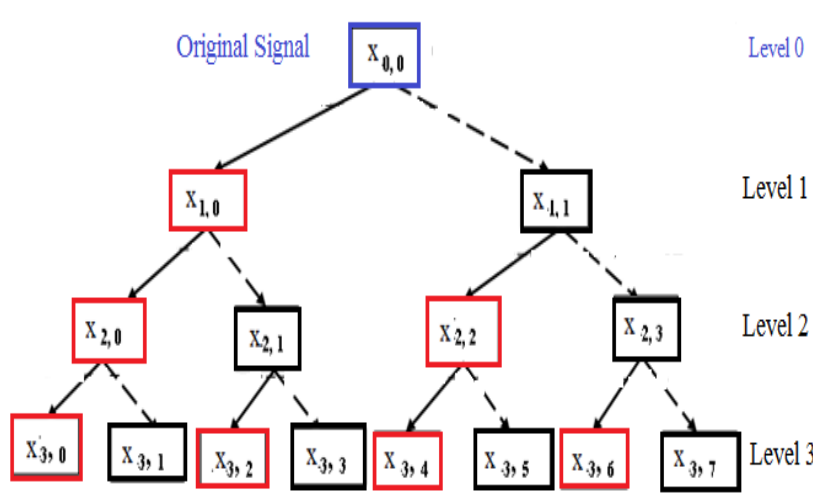

Fig. 4. Three level decomposition of vibration signal using WP

variable in order to accomplish better accuracy, avoid over fitting and decreases training and validation time. The feature subset selection techniques do not change the original data representation [3]. For this analysis, we applied the Bhattacharya space method to select top ten features on the basis of Bhattacharya space score. The Bhattacharya score is obtained by using equation (11). The Bhattacharya space of $f^{t h}$ feature in $\eta^{t h}$ class label matrix is define as

$$
B h\left(X^{J}\right)=\frac{4 *\left(\mu_{1}-\mu_{2}\right)^{2}}{\left(d_{1}+d_{2}\right)}+2 \log \left(\frac{\left(d_{1}+d_{2}\right)}{\sqrt{\frac{d_{1} * d_{2}}{2}}}\right)
$$

Where $d_{1}=\sqrt{V_{1}}, d_{2}=\sqrt{V_{2}}$ and $B h\left(X^{J}\right)$ represents Bhattacharya score of Jth feature. $\mu_{1}$ and $\mu_{2}$ mean of 1 stand 2nd group of Jth feature. $V_{1}$ and $V_{2}$ variance of 1 st and 2nd group of Jth feature. Sometimes, the features extracted from vibration signal at different bearing conditions after features ranking or selection are correlated, and therefore redundant. It must also ensure that similar information is conveyed in a concise manner. In case, the feature selection approach is not suitable to select most dissimilar features. This can be where features dimensionality reduction algorithms like GDA and KPCA inherit play. Dimension reduction mainly states to the process of converting a features dimension. GDA uses the kernel function operator to analyze nonlinear discriminant. The goal of GDA is to find out a projection for the variables into a lower dimensional size by increasing the quantitative relation between-class scatter to within-class scatter. In this paper, we have applied a Gaussian and radial basis function (RBF) kernel [19] to transform the top ten rank LWPFs to new feature.

\section{E. Gaussian support vector machine}

Support vector machine (SVM) is a supervised arithmetical learning model based on the concept of decision hyper-plane that delineate decision boundaries for solving both classification or detection and regression problem. A hyper decision plane is one that splits a set of features not having the same class labels. The nearest data points generated by SVM to optimal separating hyper decision plane are well-known as support vectors.

Assume that a training features set with class label $T=\left(a_{i}, b_{i}\right)_{i=1}^{n}$ where $a_{i} \in R^{N}$, and $b_{i} \in\left\{+1_{s}-1\right\}$, belong to two classes. The hyper decision plane employed two separate linearly trained features. Two class labels are defined as $P(a)=W^{t} a_{i}+\beta$, where $W$ represents weight vector, $\beta$ and ${ }^{t}$ denotes a scalar quantity as bias and indicates transport of $W$, in such a way that $P(a) \geq 0$ for $b_{i}=+1$ and $P(a) \leq 0$ for $b_{i}=-1$. In this case, the optimal hyper decision plane can be calculated by solving the quadratic optimization problem $i z(\eta)=\frac{1}{2}\|W\|^{2}$, with condition $b_{i}\left(W^{t} a_{i}+\beta\right) \geq 1$. If the features are nonlinearly separable then the goal of SVM is to maximize the margin between features of two class labels (separation) and reduce the errors. This process has been done by using constrained optimization problem using a transformation matrix $\Phi\left(a_{i}\right)$ [20]. In this case, optimized hyper decision plane can be evaluated by solving the equation $\operatorname{Minimiz}(\eta)=\frac{1}{2}\|W\|^{2}+C \sum_{i=1}^{n} \xi_{i}$, with condition $b_{i}\left(W^{t} \phi\left(a_{i}\right)+\beta\right) \geq 1-\xi_{i}$, where $\mathrm{C}$ denotes free error weight (regularization parameter) and it processes the size of the penalties allotted to the errors and $\bar{\xi}$ is known as slack variable and measures error of data. Therefore, hyper decision plane can be formulated as

$P(a)=\operatorname{Sign}\left\{\sum_{i=1}^{\mathbb{N}} \alpha_{i} b_{i} K\left(a_{i}, a\right)+\beta\right\}$

Where $\alpha_{i}$ represents Lagrange multiplier and $K\left(a_{i}, a\right)$ denotes kernel function. In this article, Gaussian function is applied as kernel function due to its better performance [21] The Gaussian kernel function is defined as

$K\left(a_{i}, a\right)=\operatorname{Exp}^{\left(-\frac{\left(a_{i}-a^{2}\right)^{2}}{2 \sigma^{2}}\right)}$

\section{F. Performance parameters of the model}

To analyze the performance of classifier with considered datasets, performance parameters were calculated as accuracy $(\mathrm{AC})=(\mathrm{TP}+\mathrm{TN}) /(\mathrm{TP}+\mathrm{TN}+\mathrm{FN}+\mathrm{FP})$, Sensitivity $(\mathrm{SE})$ or true positive rate $(\mathrm{TPR})=\mathrm{TP} /(\mathrm{TP}+\mathrm{FN})$ and specificity (SP) or true negative rate $(\mathrm{TNR})=\mathrm{TN} /(\mathrm{FP}$ $+\mathrm{TN})$ and false positive rate $(\mathrm{FPR})=(1-\mathrm{SP})$ using confusion matrix. Where $\mathrm{TP}=$ true positive, $\mathrm{FN}=$ false negative, $\mathrm{TN}=$ true negative and $\mathrm{FP}=$ false positive.

The generalization ability of model depends on three parameters ${ }^{C},{ }^{\sigma}$ and $\xi$ [21]. Where ${ }^{\sigma}$ denotes the width of Gaussian kernel function, $C$ is regularization parameter for optimized hyper decision plane (which deals with trade-off / error between training error and the smoothness of the output solution) and $\xi$ provides measurement of error data in hyper plane. Accurate classification of training datasets depends on selection value of $C$. If the value of $C$ is large, optimization will take a less-margin hyper decision plane to train data properly and vice versa for very small value. To overcome the selection of trial value of $C$, we have optimized this parameter by applying ten cross validation approach. The classification performances in terms of $A C$, $S E$ and $S P$ for training and validation of model were evaluated using ten trials with ten-folds cross validation procedure, in which each trial data sets were randomly divided into 5 parts to validate the robustness of Model. It is standard technique to validate any model. 


\section{SIMULATION AND RESULTS}

In this paper, database of thirty six vibration signal produced by rolling bearing as reported in Table 1 are taken. These database are associated with running states of various bearing conditions as no fault (NF), inner-ring wear (IR) and lack of ball bearings (BB) faults. These database were analyzed using decomposed vibration signal by WP methods and GSVM classifier. Each database were investigated on different loads $0 \mathrm{HP}, 1 \mathrm{HP}, 2 \mathrm{HP}$ and $3 \mathrm{HP}$. The simulation results were studied by relating NF with IR7, IR14, IR21, IR28, and BB7, BB14, BB21 and BB28 datasets. For this analysis, five level WP was taken to decompose the vibration signal into sixty three approximation (Apx.) and detail (Det.) frequency range. With the aim to improve the classification performance and features difference capability, the Logarithmic Wavelet Packet Features (LWPFs) were extracted from decomposed vibration signals. To suppress the redundant features and avoid overfitting of classification, Bhattacharya space feature selection method is added to rank the appropriate features. The top ten features (highest score) were taken out for analysis of bearing faults. Achieved score associated to each database is reported in Table II, III, IV and V. The numerical results of simulation reveals that 5 th levels decomposed features (62LWPFS-L5-Apx) have the highest score for each database compared to fourth level decomposition of databases. From this, we observed that 5th levels decomposed features have highest mean and lowest variance (from equation 11) and these features are more effective compared to 1 st, 2 nd and 3rd level decomposition for detection of bearing faults. It happens due

Table II

Bhattacharya Score Of Top 10 Features Of Nf-Ir7 And Nf-Ir14 Data Set Arranged In Descending Order

\begin{tabular}{|c|c|c|c|}
\hline Feature & NF-IR7 & Feature & NF-IR14 \\
\hline 62-LWPFS-L5-Apx. & 886.125 & 62-LWPFS-L5-Apx. & 713.153 \\
\hline 58-LWPFS-L5-Apx. & 782.482 & 60-LWPFS-L5-Apx. & 651.327 \\
\hline 63-LWPFS-L5-Del. & 624.632 & 58-LWPFS-L5-Apx. & 510.132 \\
\hline 52-LWPFS-L5-Apx. & 604.114 & 63-LWPFS-L5-Del. & 478.007 \\
\hline 61-LWPFS-L5-Del. & 589.812 & 56-LWPFS-L5-Apx. & 312.143 \\
\hline 51-LWPFS-L5-Del. & 498.234 & 48-LWPFS-L5-Apx. & 286.014 \\
\hline 56-LWPFS-L5-Apx. & 432.321 & 28-LWPFS-L4-Apx. & 188.239 \\
\hline 43-LWPFS-L5-Del. & 405.122 & 59-LWPFS-L5-Del. & 169.489 \\
\hline 38-LWPFS-L5-Apx. & 324.151 & 49-LWPFS-L5-Del. & 122.415 \\
\hline 41-LWPFS-L5-Del. & 314.196 & 52-LWPFS-L5-Apx. & 96.103 \\
\hline
\end{tabular}

Table III

Bhattacharya Score Of Top 10 Features Of Nf-Ir21 And Nf-Ir28 Data Set Arranged In Descending Order

\begin{tabular}{|c|c|c|c|}
\hline Feature & NF-IR21 & Feature & NF-IR28 \\
\hline 62-LWPFS-L5-Apx. & 228.122 & 62-LWPFS-L5-Apx. & 1120.413 \\
\hline 60-LWPFS-L5-Apx. & 218.712 & 58-LWPFS-L5-Apx. & 1001.245 \\
\hline 58-LWPFS-L5-Apx. & 196.437 & 56-LWPFS-L5-Apx. & 989.183 \\
\hline 63- LWPFS-L5-Del. & 171.326 & 42-LWPFS-L5-Apx. & 843.532 \\
\hline 28-LWPFS-L4-Apx. & 163.421 & 63-LWPFS-L5-Del. & 784.612 \\
\hline 49- LWPFS-L5-Del. & 152.131 & 25-LWPFS-L4-Del. & 683.749 \\
\hline 21-LWPFS-L4-Del. & 111.162 & 48-LWPFS-L5-Apx. & 645.153 \\
\hline
\end{tabular}

to high resolution of Apx. And Det. frequency at 5th level features.

In each database of top ten features, there is a large gap in features score. This indicates that these features are appropriate to discriminate the healthy and non-healthy bearing. In order to analyze variation in datasets of bearing faults with respect to healthy bearing, we have evaluated mean and standard deviation (SD) parameters of top ten LWPFS features in time domain. These parameters are reported in Table VI, VII, VIII and IX. The results of table illustrate that mean value of top ten features obtained from NF bearing is higher than faulty bearings. This table also shows that mean value of top ten

TABLE IV

BHATTACHARYA SCORE OF TOP 10 FEATURES OF NF-BB7 AND NF-BB14 DATA SET ARRANGED IN DESCENDING ORDER

\begin{tabular}{|c|c|c|c|}
\hline Feature & NF-BB7 & Feature & NF-BB14 \\
\hline 62-LWPFS-L5-Apx. & 657.142 & 62-LWPFS-L5-Apx. & 612.115 \\
\hline 58-LWPFS-L5-Apx. & 612.341 & 58-LWPFS-L5-Apx. & 601.342 \\
\hline 56-LWPFS-L5-Apx. & 588.936 & 54-LWPFS-L5-Apx. & 589.427 \\
\hline 51-LWPFS-L5-Del. & 536.673 & 51-LWPFS-L5-Del. & 574.497 \\
\hline 52-LWPFS-L5-Apx. & 429.434 & 38-LWPFS-L5-Apx. & 544.327 \\
\hline 54-LWPFS-L5-Apx. & 436.342 & 29-LWPFS-L4-Del. & 498.461 \\
\hline 37-LWPFS-L5-Del. & 403.243 & 63-LWPFS-L5-Del. & 435.315 \\
\hline 57-LWPFS-L5-Del. & 389.146 & 39-LWPFS-L5-Del. & 387.245 \\
\hline 43-LWPFS-L5-Del. & 236.004 & 59-LWPFS-L5-Del. & 298.164 \\
\hline 39-LWPFS-L5-Del. & 214.491 & 36-LWPFS-L5-Apx. & 276.322 \\
\hline
\end{tabular}

Table V

Bhattacharya Score Of Top 10 Features Of Nf-Bb21 And Nf-Bb28 Data Set Arranged In Descending Order

\begin{tabular}{|c|c|c|c|}
\hline Feature & NF-BB21 & Feature & NF-BB28 \\
\hline 62-LWPFS-L5-Apx. & 1167.214 & 62-LWPFS-L5-Apx. & 126.103 \\
\hline 52-LWPFS-L5-Apx. & 1059.248 & 52-LWPFS-L5-Del. & 121.124 \\
\hline 58-LWPFS-L5-Apx. & 1018.121 & 56-LWPFS-L5-Apx. & 101.121 \\
\hline 63-LWPFS-L5-Del. & 987.430 & 58-LWPFS-L5-Apx. & 89.034 \\
\hline 48-LWPFS-L5-Apx. & 981.324 & 63-LWPFS-L5-Del. & 81.843 \\
\hline 39-LWPFS-L5-Del. & 802.234 & 43-LWPFS-L5-Del. & 79.462 \\
\hline 54-LWPFS-L5-Apx. & 788.124 & 36-LWPFS-L5-Apx. & 71.412 \\
\hline 29-LWPFS-L4-Del. & 685.345 & 30-LWPFS-L4-Apx. & 69.356 \\
\hline 51-LWPFS-L5-Del. & 603.214 & 33-LWPFS-L5-Del. & 61.129 \\
\hline 46-LWPFS-L5-Apx. & 588.131 & 48-LWPFS-L5-Apx. & 58.402 \\
\hline
\end{tabular}

LWPFS of IR bearing defect is higher than that of ball BB faults. The results of Table VI, VII, VIII and IX also illustrate that the SD of top ten features of NF data is lower than IR7, IR14, IR21, IR28 and BB7, BB14, BB21, BB28 bearing. It reflects that signal generated by healthy bearing is lees affected by noise compared to faulty bearing. 
Besides stastical measurement, GSVM with several dimension reduction schemes having different kernel functions were employed in this paper to attain maximum validation accuracy (AC) by least number of LWPFS features out of top ten features. Top ten features taken from LWPFS decomposed vibration signal were structured according to their rank score of Bhattacharya space. The rank features (highest to lowest score) were fed to the GSVM one by one until the maximum

Table.Vi

Mean Of Top 10 Features Ranked By Bhattacharya Space Of Considered Datasets

\begin{tabular}{|c|c|c|c|c|}
\hline NF & IR7 & IRl4 & IR21 & IR28 \\
\hline mean & mean & mean & mean & mean \\
\hline-3.3423 & -0.7923 & -1.3461 & 0.0934 & -0.6941 \\
\hline-2.1341 & -0.7845 & -1.6814 & -0.9846 & -0.1683 \\
\hline-2.6478 & -1.0453 & -1.2443 & 0.0978 & -0.0047 \\
\hline-2.8764 & -1.6723 & -1.5438 & -0.7564 & -0.1976 \\
\hline-1.4356 & -0.8941 & -1.3421 & -1.3402 & -0.3941 \\
\hline-1.4536 & -1.0673 & -1.4236 & -0.4782 & -0.0324 \\
\hline-2.0764 & -0.6743 & -1.9878 & -0.6521 & -0.4356 \\
\hline-1.7456 & -0.7843 & -1.0437 & -0.3451 & -0.0436 \\
\hline-1.3456 & -1.0432 & -1.7342 & -0.3945 & -0.1743 \\
\hline-2.4532 & -0.8742 & -2.5427 & -0.1467 & -1.3464 \\
\hline
\end{tabular}

Table.Vii

Mean Of Top 10 Features Ranked By Bhattacharya Space Of Considered Datasets

\begin{tabular}{|c|c|c|c|c|}
\hline NF & BB7 & BB14 & BB21 & BB28 \\
\hline mean & mean & mean & mean & mean \\
\hline-3.3423 & -1.0431 & -1.0342 & -.9821 & -1.4921 \\
\hline-2.1341 & -1.8934 & -1.4861 & -1.4128 & -0.6921 \\
\hline-2.6478 & -1.3421 & -1.4521 & -1.5361 & -0.9832 \\
\hline-2.8764 & -1.4231 & -2.4341 & -2.0342 & -0.0981 \\
\hline-1.4356 & -1.9834 & -1.7935 & -2.4382 & -1.0532 \\
\hline-1.4536 & -1.3045 & -2.0211 & -2.0231 & -0.8721 \\
\hline-2.0764 & -1.9211 & -1.4821 & -1.5923 & -1.5942 \\
\hline-1.7456 & -1.4324 & -1.7643 & -1.8432 & -0.6934 \\
\hline-1.3456 & -1.5403 & -1.7943 & -1.4324 & -0.8721 \\
\hline-2.4532 & -1.3424 & -3.1241 & -2.9876 & -0.5932 \\
\hline
\end{tabular}

Performance as AC was obtained.

A graph with number of LWPFS features versus validation accuracies using different classification approaches are shown in Fig. 5 (a) and (b) for NF-IR14 and NF-BB14 dataset.

In Fig. 5 (a), the proposed GSVM classifier achieved 100 $\%$ validation AC for two top LWPFS features only for NFIR14 dataset.

In Fig. 5 (b), SVM using multi quadric and sigmoid hidden node with GDA and Gaussian kernel achieved maximum validation AC of $100 \%$ for every top ten LWPFS features (except two features).
Table.Viii

Standard Deviation $( \pm$ Sd) Of Top 10 Features Ranked By Bhattacharya Space Of Considered Datasets

\begin{tabular}{|c|c|c|c|c|}
\hline $\mathbf{N F}$ & $\mathbf{I R 7}$ & $\mathbf{I R 1 4}$ & $\mathbf{I R 2 1}$ & $\mathbf{I R 2 8}$ \\
\hline $\mathbf{\pm S D}$ & $\mathbf{\pm S D}$ & $\mathbf{\pm S D}$ & $\mathbf{\pm S D}$ & $\mathbf{\pm S D}$ \\
\hline 0.0143 & 0.0451 & 0.0352 & 0.0891 & 0.0365 \\
\hline 0.0325 & 0.0523 & 0.0435 & 0.0791 & 0.0426 \\
\hline 0.0294 & 0.0382 & 0.0642 & 0.0671 & 0.0534 \\
\hline 0.0298 & 0.0513 & 0.0158 & 0.0782 & 0.0436 \\
\hline 0.0453 & 0.0412 & 0.0867 & 0.0983 & 0.0642 \\
\hline 0.0342 & 0.0264 & 0.9843 & 0.0794 & 0.0602 \\
\hline 0.0145 & 0.0432 & 0.0891 & 0.0865 & 0.0732 \\
\hline 0.0045 & 0.0563 & 0.0756 & 0.0843 & 0.0472 \\
\hline 0.0161 & 0.0124 & 0.0482 & 0.0745 & 0.0263 \\
\hline 0.0074 & 0.0431 & 0.1423 & 0.0976 & 0.0352 \\
\hline
\end{tabular}

Table.Ix

Standard Deviation $( \pm$ Sd) Of Top 10 Features Ranked By Bhattacharya Space Of Considered Datasets

\begin{tabular}{|c|c|c|c|c|}
\hline $\mathbf{N F}$ & $\mathbf{B B 7}$ & $\mathbf{B B 1 4}$ & $\mathbf{B B 2 1}$ & $\mathbf{B B 2 8}$ \\
\hline $\mathbf{\pm S D}$ & $\mathbf{\pm S D}$ & $\mathbf{\pm S D}$ & $\mathbf{\pm} \mathbf{S D}$ & $\mathbf{\pm S D}$ \\
\hline 0.0143 & 0.0431 & 0.0452 & 0.0432 & 0.0532 \\
\hline 0.0325 & 0.0392 & 0.0352 & 0.0412 & 0.0492 \\
\hline 0.0294 & 0.0432 & 0.0342 & 0.0542 & 0.0392 \\
\hline 0.0298 & 0.0643 & 0.0762 & 0.0342 & 0.0421 \\
\hline 0.0453 & 0.0693 & 0.0357 & 0.0546 & 0.0392 \\
\hline 0.0342 & 0.0763 & 0.0321 & 0.0634 & 0.0392 \\
\hline 0.0145 & 0.0436 & 0.3462 & 0.0982 & 0.0491 \\
\hline 0.0045 & 0.0467 & 0.0421 & 0.0673 & 0.0392 \\
\hline 0.0161 & 0.0692 & 0.0352 & 0.0412 & 0.0631 \\
\hline 0.0074 & 0.06942 & 0.0453 & 0.0043 & 0.0526 \\
\hline
\end{tabular}

From Fig. 5 (a) and (b), we observed that when SVM is used with GDA, the faults detection $A C$ increases compared to SVM with LDA and SVM with KPCA. These results clearly indicate the importance of GDA in fault detection $A C$, when it was applied as a feature space transformation technique.

In order to test the bearing faults detection performance of proposed automated approach, the classification performance were calculated in terms of $\mathrm{AC}, \mathrm{SE}, \mathrm{SP}$ and PPV. 


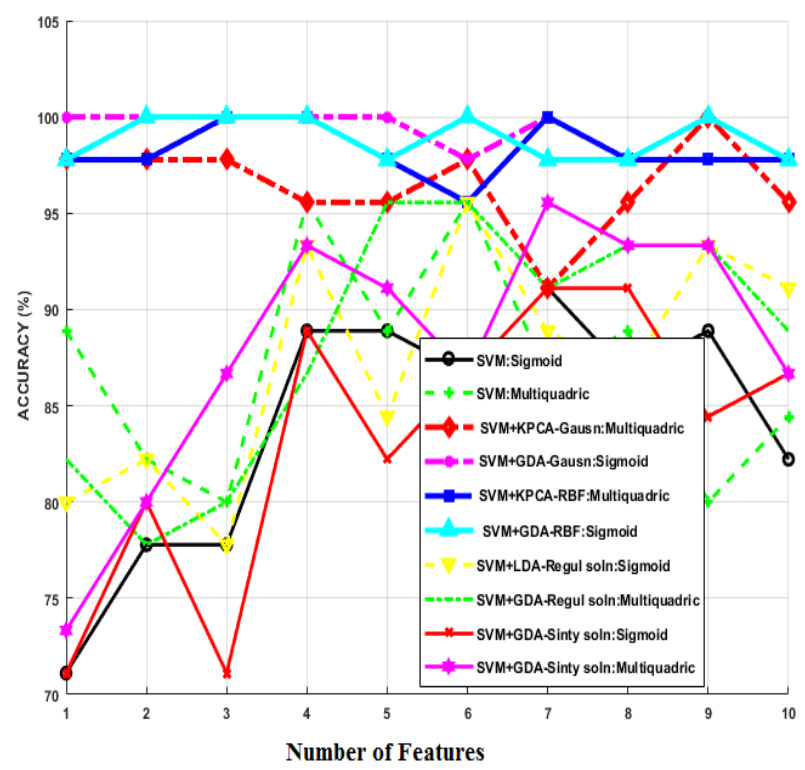

Fig. 5 (a). Plots representing detection accuracies for different number of top ten ranked features using various techniques for NF-IR14 dataset

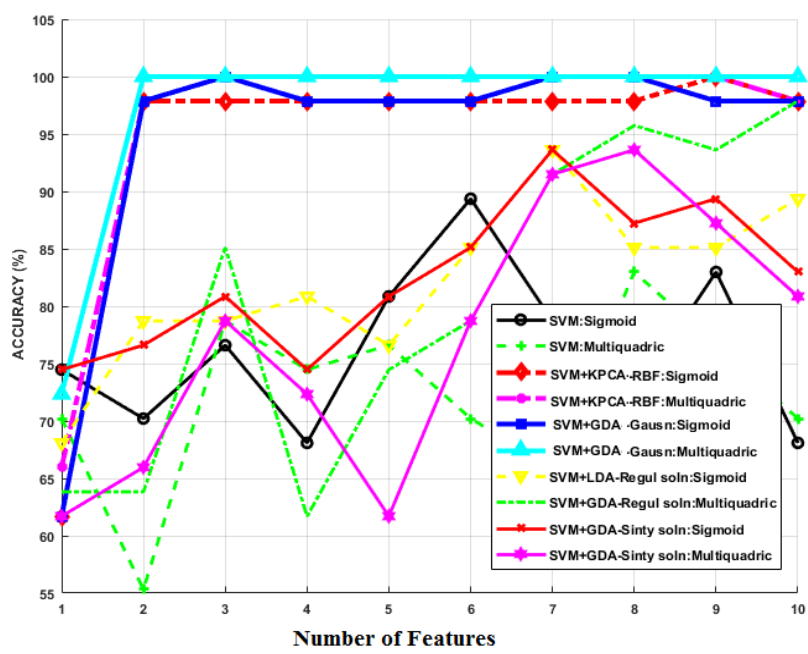

Fig. 5 (b). Plots representing detection accuracies for different number of top ten ranked features using various techniques for NF-BB14 dataset

To validate the robustness of our proposed scheme each datasets were randomly allocated as 100 out of 160 for training and remaining datasets for validation. The training and validation samples of data sets were used for simulation and achieved classifier performance are reported in Table X, XI, XII, XIII, and XIV.

\section{Table X}

Performance Investigation Of Gsvm Classifier For Bearing Fault Detection

\begin{tabular}{|c|c|c|c|c|c|c|c|c|}
\hline \multirow{2}{*}{ Datasets } & \multicolumn{9}{|c|}{ GSVM } \\
\cline { 2 - 9 } & \multicolumn{9}{|c|}{ Training } & \multicolumn{4}{c|}{ Validation } \\
\cline { 2 - 10 } & AC & SE & SP & PPV & AC & SE & SP & PPV \\
\hline NF-IR7 & 98 & 88 & 94 & 89 & 90 & 81 & 83 & 84 \\
\hline NF-IR14 & 95 & 89 & 86 & 93 & 92 & 85 & 84 & 89 \\
\hline NF-IR21 & 97 & 90 & 91 & 88 & 91 & 89 & 87 & 86 \\
\hline NF-IR28 & 92 & 93 & 94 & 95 & 88 & 90 & 89 & 87 \\
\hline NF-BB7 & 94 & 93 & 95 & 96 & 90 & 91 & 90 & 91 \\
\hline NF-BB14 & 93 & 92 & 94 & 92 & 93 & 90 & 91 & 91 \\
\hline NF-BB21 & 94 & 94 & 90 & 95 & 92 & 91 & 87 & 93 \\
\hline NF-BB28 & 90 & 90 & 93 & 91 & 87 & 90 & 89 & 91 \\
\hline
\end{tabular}

Table Xi

Performance Investigation Of Gsvm Classifier With Kpca And Rbf Kernel For Bearing Fault Detection

\begin{tabular}{|c|c|c|c|c|c|c|c|c|}
\hline \multirow{2}{*}{ Datasets } & \multicolumn{6}{|c|}{ [(KPCA and RBF kernel)+(GSVM)] } \\
\cline { 2 - 10 } & \multicolumn{4}{|c|}{ Training } & \multicolumn{5}{c|}{ Validation } \\
\cline { 2 - 10 } & AC & SE & SP & PPV & AC & SE & SP & PPV \\
\hline NF-IR7 & 95 & 92 & 95 & 92 & 92 & 89 & 92 & 89 \\
\hline NF-IR14 & 98 & 95 & 96 & 94 & 94 & 93 & 93 & 91 \\
\hline NF-IR21 & 96 & 94 & 96 & 95 & 93 & 89 & 93 & 92 \\
\hline NF-IR28 & 98 & 94 & 97 & 97 & 95 & 91 & 92 & 94 \\
\hline NF-BB7 & 97 & 97 & 98 & 99 & 94 & 93 & 94 & 97 \\
\hline NF-BB14 & 93 & 95 & 96 & 98 & 91 & 92 & 92 & 95 \\
\hline NF-BB21 & 98 & 99 & 96 & 94 & 95 & 90 & 93 & 92 \\
\hline NF-BB28 & 97 & 96 & 96 & 98 & 93 & 93 & 92 & 95 \\
\hline
\end{tabular}

The results of Table XI demonstrate that GSVM with KPCA and radial basis function (RBF) achieved classification performance as AC: 91 to 95 , SE: 89 to 93, SP: 92 to 94 and PPV: 89 to 97 for considered datasets. While results of Table XII demonstrate that GSVM with KPCA and Gaussian kernel achieved classification performance as AC: 94 to 98 , SE: 87 to 96, SP: 88 to 97 and PPV: 93 to 98 .

The results of Table XI, and XII shows that classification performance of GSVM with KPCA and Gaussian kernel is better than GSVM with KPCA and radial basis function.

The result of Table XIII shows that GSVM with GDA and RBF kernel gives classification performance parameters as AC: 97 to 98, SE: 97 to 98, SP: 96 to 100, and PPV: 97 to 99.

The results of Table XV show that when GSVM is combined with GDA and Gaussian kernel, classification performance significantly improves and became AC: 99 to 100, SE: 99 to 100, SP: 99 to 100 , and 99 to 100

Table Xii

Performance Investigation Of Gsvm Classifier With Kpca

And Gaussian Kernel For Bearing Fault Detection

\begin{tabular}{|c|c|c|c|c|c|c|c|c|}
\hline \multirow{2}{*}{ Datasets } & \multicolumn{5}{|c|}{ [(KPCA and Gaussian kernel) +(GSVM)] } \\
\cline { 2 - 10 } & \multicolumn{3}{|c|}{ Training } & \multicolumn{5}{c|}{ Validation } \\
\cline { 2 - 10 } & AC & SE & SP & PPV & AC & SE & SP & PPV \\
\hline NF-IR7 & 100 & 100 & 98 & 100 & 98 & 93 & 95 & 98 \\
\hline NF-IR14 & 96 & 90 & 99 & 96 & 95 & 87 & 94 & 93 \\
\hline NF-IR21 & 97 & 96 & 97 & 98 & 93 & 90 & 95 & 94 \\
\hline NF-IR28 & 99 & 97 & 98 & 99 & 94 & 92 & 91 & 94 \\
\hline NF-BB7 & 97 & 100 & 100 & 99 & 94 & 93 & 88 & 97 \\
\hline NF-BB14 & 100 & 99 & 99 & 96 & 98 & 91 & 96 & 95 \\
\hline NF-BB21 & 99 & 98 & 98 & 97 & 95 & 92 & 97 & 94 \\
\hline NF-BB28 & 98 & 99 & 100 & 99 & 98 & 96 & 93 & 96 \\
\hline
\end{tabular}


Table XIII

Performance Investigation Of Gsvm Classifier With Gda And Rbf Kernel For Bearing Fault Detection.

\begin{tabular}{|c|c|c|c|c|c|c|c|c|}
\hline \multirow{3}{*}{ Datasets } & \multicolumn{5}{|c|}{ (GDA and RBF kernel) + (GSVM) } \\
\cline { 2 - 10 } & \multicolumn{3}{|c|}{ Training } & \multicolumn{5}{c|}{ Validation } \\
\cline { 2 - 10 } & $A C$ & $S E$ & $S P$ & $P P V$ & $A C$ & $S E$ & $S P$ & $P P V$ \\
\hline NF-IR7 & 100 & 97 & 99 & 98 & 97 & 97 & 96 & 97 \\
\hline NF-IR14 & 99 & 100 & 99 & 100 & 98 & 97 & 98 & 98 \\
\hline NF-IR21 & 100 & 98 & 99 & 100 & 98 & 97 & 99 & 99 \\
\hline NF-IR28 & 100 & 98 & 98 & 98 & 97 & 98 & 97 & 98 \\
\hline NF-BB7 & 100 & 97 & 99 & 98 & 98 & 97 & 97 & 96 \\
\hline NF-BB14 & 100 & 99 & 100 & 100 & 98 & 97 & 100 & 99 \\
\hline NF-BB21 & 99 & 98 & 100 & 99 & 98 & 97 & 98 & 98 \\
\hline NF-BB28 & 100 & 99 & 99 & 100 & 98 & 98 & 97 & 98 \\
\hline
\end{tabular}

From the results of Table X, XI, XII, XIII, and XIV it can be concluded that the performance of GSVM with GDA and Gaussian kernel function has a higher performance rate. This is due to the combination of GSVM with Bhattacharya space and GDA as features dimensions transformation with appropriate kernel.

\section{DISCUSSIONS}

In recent works done by authors shown in Table XV, authors have not considered non-linear features to detect the faults of bearing. Our proposed method consists of nonlinear LWPFS features, dimension reduction technique GDA with Gaussian kernel function and GSVM classifier. Our proposed method produced classification accuracy of $100 \%$ for IR and BB faults.

Table Xiv

Performance Investigation Of Gsvm Classifier With Gda And Gaussian Kernel For Bearing Fault Detection.

\begin{tabular}{|l|c|c|c|c|c|c|c|c|}
\hline \multirow{3}{*}{ Datasets } & \multicolumn{4}{|c|}{ (GDA and Gaussian kernel) + (GSVM) } \\
\cline { 2 - 9 } & \multicolumn{3}{|c|}{ Training Performance } & \multicolumn{4}{c|}{ Validation } \\
\cline { 2 - 9 } & AC & SE & $S P$ & $P P V$ & $A C$ & $S E$ & $S P$ & $P P V$ \\
\hline NF-IR7 & 100 & 100 & 100 & 100 & 100 & 100 & 98 & 99 \\
\hline $\begin{array}{l}\text { NF- } \\
\text { IR14 }\end{array}$ & 100 & 100 & 100 & 100 & 100 & 100 & 99 & 99 \\
\hline $\begin{array}{l}\text { NF- } \\
\text { IR21 }\end{array}$ & 100 & 100 & 100 & 100 & 100 & 99 & 99 & 99 \\
\hline $\begin{array}{l}\text { NF- } \\
\text { IR28 }\end{array}$ & 100 & 100 & 100 & 100 & 99 & 100 & 99 & 99 \\
\hline $\begin{array}{l}\text { NF- } \\
\text { BB7 }\end{array}$ & 100 & 100 & 100 & 100 & 99 & 100 & 99 & 99 \\
\hline $\begin{array}{l}\text { NF- } \\
\text { BB14 }\end{array}$ & 100 & 100 & 100 & 100 & 100 & 100 & 100 & 100 \\
\hline $\begin{array}{l}\text { NF- } \\
\text { BB21 }\end{array}$ & 100 & 100 & 100 & 100 & 100 & 99 & 100 & 99 \\
\hline $\begin{array}{l}\text { NF- } \\
\text { BB28 }\end{array}$ & 100 & 100 & 100 & 100 & 100 & 99 & 99 & 100 \\
\hline
\end{tabular}

Table XV

Recent Work Done By Authors For Investigations And Detection Of Bearing Faults

\begin{tabular}{|c|c|c|c|c|}
\hline $\begin{array}{l}\text { Reference } \\
\text { Number }\end{array}$ & $\begin{array}{c}\text { Feature } \\
\text { Extraction } \\
\text { Method }\end{array}$ & $\begin{array}{c}\text { Feature } \\
\text { Reduction } \\
\text { Method }\end{array}$ & $\begin{array}{c}\text { Classification } \\
\text { Technique }\end{array}$ & $\begin{array}{c}\text { Classification } \\
\text { Accuracy } \\
(\%)\end{array}$ \\
\hline [14] & DMW & LDA & SVM & 100 \\
\hline [15] & CMW & PCA & SVM & 96.67 \\
\hline [17] & $\begin{array}{c}10 \text { statistical } \\
\text { and } 3 \mathrm{FD}\end{array}$ & PCA & SVM & 98.1 \\
\hline [18] & TD Features & LDA & SVM & 98.94 \\
\hline [20] & TD Features & KPCA & SVM & 96.33 \\
\hline [37] & EEMD & Filter Ranking & SVM & 100 \\
\hline [38] & WPT \& MPE & Fisher Score & HMM & 94.2 \\
\hline [39] & MPE & GDA & SVM & 99.89 \\
\hline [40] & FB & GDA & SFAM & 100 \\
\hline [41] & WPT & PCA & ANFIS & 99.8 \\
\hline [42] & $\begin{array}{c}\text { Image of } \\
\text { vibration } \\
\text { signal }\end{array}$ & GDA & $\mathrm{CNN}$ & 99.75 \\
\hline [43] & $\begin{array}{l}\text { Visualizing } \\
\text { data using t- } \\
\text { SNE }\end{array}$ & NA & DGNN & 97.81 \\
\hline [44] & $\mathrm{VMD}$ & PCA & $\begin{array}{c}\text { SVM with } \\
\text { Polynomial, } \\
\text { Pearson } \\
\text { Universal Kernel } \\
\text { (PUK) and RBF } \\
\text { kernel }\end{array}$ & $\begin{array}{c}\text { Polynomial: } \\
\quad 93.733 \\
\text { PUK: } 99.28 \\
\text { RBF: } 80.22\end{array}$ \\
\hline [45] & $\begin{array}{l}\text { Multiple } \\
\text { Swarm }\end{array}$ & NA & $\begin{array}{c}\text { Genetic } \\
\text { Algorithm and } \\
\text { SVM }\end{array}$ & 93.33 \\
\hline [46] & $\begin{array}{l}\text { Compressed } \\
\text { sensing (CS) }\end{array}$ & NA & Deep learning & $97.47( \pm 0.43)$ \\
\hline [47] & TD Features & NA & SVM & 99.2 \\
\hline $\begin{array}{l}\text { Proposed } \\
\text { Work }\end{array}$ & $\begin{array}{c}\text { WP } \\
\text { decomposition }\end{array}$ & $\begin{array}{c}\text { Bhattacharya } \\
\text { Score and } \\
\text { GDA }\end{array}$ & GSVM & 100 \\
\hline
\end{tabular}

\section{CONCLUSION}

In this article, a novel automated and appropriate bearing fault detection approach is presented. This is based on the combination of GSVM, Bhattacharya space and features dimension reduction scheme. For this, LWPFS features are extracted from non-linear vibration signal. The MATLAB simulated results have shown that minor fluctuation in vibration signal can be identified. On the basis of data augmentation of mean and SD value of top ten LWPFS, NF bearing has high mean and low SD compared to IR and BB faults. Numerical Results of Table IV shows that proposed model could achieve excellent classification performance when using GSVM and GDA with Gaussian kernel. Form above results, it is concluded that proposed automated approach can be applied to address the diagnosis and analysis of bearing faults.

\section{REFERENCES}

S. Nandi, H. A. Toliyat, and X. Li, "Condition monitoring and faul diagnosis of electrical motors - A review," IEEE Transactions on Energy Conversion, vol. 20, no. 4. pp. 719-729, 2005.

2. N. Tandon, G. S. Yadava, and K. M. Ramakrishna, "A comparison of some condition monitoring techniques for the detection of defect in induction motor ball bearings," Mech. Syst. Signal Process., vol. 21 , no. 1, pp. 244-256, 2007.

3. P. Zhang, Y. Du, T. G. Habetler, and B. Lu, "A survey of condition monitoring and protection methods for medium-voltage induction motors," IEEE Transactions on Industry Applications, vol. 47, no. 1 pp. 34-46, 2011.

4. M. Blodt, P. Granjon, B. Raison, and G. Rostaing, "Models for bearing damage detection in induction motors using stator current monitoring," IEEE Trans. Ind. Electron., vol. 55, no. 4, pp. 18131822,2008 
5. F. Immovilli, A. Bellini, R. Rubini, and C. Tassoni, "Diagnosis of bearing faults in induction machines by vibration or current signals: A critical comparison,” IEEE Trans. Ind. Appl., vol. 46, no. 4, pp. 1350$1359,2010$.

6. C. Ruiz-Cárcel, V. H. Jaramillo, D. Mba, J. R. Ottewill, and Y. Cao, "Combination of process and vibration data for improved condition monitoring of industrial systems working under variable operating conditions," Mech. Syst. Signal Process., vol. 66-67, pp. 699-714, 2016.

7. H. Qiu, J. Lee, J. Lin, and G. Yu, "Wavelet filter-based weak signature detection method and its application on rolling element bearing prognostics," J. Sound Vib., vol. 289, no. 4-5, pp. 1066 1090, 2006.

8. R. Z. Xu, L. Xie, M. C. Zhang, and C. X. Li, "Machine degradation analysis using fuzzy CMAC neural network approach," Int. J. Adv. Manuf. Technol., vol. 36, no. 7-8, pp. 765-772, 2008

9. G. Yu, C. Li, and J. Sun, "Machine fault diagnosis based on Gaussian mixture model and its application,” Int. J. Adv. Manuf. Technol., vol. 48, no. 1-4, pp. 205-212, 2010.

10. Y. N. Pan, J. Chen, and G. M. Dong, "A hybrid model for bearing performance degradation assessment based on support vector data description and fuzzy c-means," Proc. Inst. Mech. Eng. Part C J. Mech. Eng. Sci., vol. 223, no. 11, pp. 2687-2695, 2009.

11. N. E. Huang et al., "The empirical mode decomposition and the Hubert spectrum for nonlinear and non-stationary time series analysis,” Proc. R. Soc. A Math. Phys. Eng. Sci., vol. 454, no. 1971 , pp. 903-995, 1998

12. Y. Wang, S. Kang, Y. Jiang, G. Yang, L. Song, and V. I. Mikulovich, "Classification of fault location and the degree of performance degradation of a rolling bearing based on an improved hyper-spherestructured multi-class support vector machine," in Mechanical Systems and Signal Processing, 2012, vol. 29, pp. 404-414.

13. O. Rioul and M. Vetterli, "Wavelets and Signal Processing," IEEE Signal Process. Mag., vol. 8, no. 4, pp. 14-38, 1991

14. S. Abbasion, A. Rafsanjani, A. Farshidianfar, and N. Irani, "Rolling element bearings multi-fault classification based on the wavelet denoising and support vector machine," Mech. Syst. Signal Process., vol. 21, no. 7, pp. 2933-2945, 2007.

15. P. Konar and P. Chattopadhyay, "Bearing fault detection of induction motor using wavelet and Support Vector Machines (SVMs)," Appl. Soft Comput. J., vol. 11, no. 6, pp. 4203-4211, 2011.

16. H. Erişti, A. Uçar, and Y. Demir, "Wavelet-based feature extraction and selection for classification of power system disturbances using support vector machines," Electr. Power Syst. Res., vol. 80, no. 7, pp 743-752, 2010.

17. T. W. Rauber, F. De Assis Boldt, and F. M. Varejão, "Heterogeneous feature models and feature selection applied to bearing fault diagnosis," IEEE Trans. Ind. Electron., vol. 62, no. 1, pp. 637-646, 2015 .

18. B. R. Nayana and P. Geethanjali, "Analysis of Statistical TimeDomain Features Effectiveness in Identification of Bearing Faults from Vibration Signal,” IEEE Sens. J., vol. 17, no. 17, pp. 56185625, 2017.

19. C. C. Reyes-Aldasoro and A. Bhalerao, "The Bhattacharyya space for feature selection and its application to texture segmentation," Pattern Recognit., vol. 39, no. 5, pp. 812-826, 2006.

20. C. Wang, L. M. Jia, and X. F. Li, Fault Diagnosis Method for the Train Axle Box Bearing Based on KPCA and GA-SVM, vol. 441 2013.

21. F. Deng, S. Yang, Y. Liu, Y. Liao, and B. Ren, "Fault Diagnosis of Rolling Bearing Using the Hermitian Wavelet Analysis, KPCA and SVM," in Proceedings - 2017 International Conference on Sensing, Diagnostics, Prognostics, and Control, SDPC 2017, 2017, vol. 2017 December, pp. 632-637.

22. K. A. Loparo, "Case Western Reserve University Bearing Data Center," Bearings Vibration Data Sets, Case Western Reserve University:http://csegroups.case.edu/bearingdatacenter/home, 2012.

23. W. Caesarendra and T. Tjahjowidodo, "A Review of Feature Extraction Methods in Vibration-Based Condition Monitoring and Its Application for Degradation Trend Estimation of Low-Speed Slew Bearing," Machines, vol. 5, no. 4, p. 21, 2017.

24. Y. Wang, G. Xu, L. Liang, and K. Jiang, "Detection of weak transient signals based on wavelet packet transform and manifold learning for rolling element bearing fault diagnosis," Mech. Syst. Signal Process. vol. 54, no. 3, pp. 259-276, 2015 .

25. L. Eren and M. J. Devaney, "Bearing Damage Detection via Wavelet Packet Decomposition of the Stator Current," IEEE Trans. Instrum. Meas., vol. 53, no. 2, pp. 431-436, 2004.

26. R. N. Khushaba, S. Kodagoda, S. Lal, and G. Dissanayake, "Driver drowsiness classification using fuzzy wavelet-packet-based feature- extraction algorithm,” IEEE Trans. Biomed. Eng., vol. 58, no. 1, pp. 121-131, 2011.

27. I. Guyon, J. Weston, S. Barnhill, and V. Vapnik, "Gene selection for cancer classification using support vector machines," Mach. Learn., vol. 46, no. 1-3, pp. 389-422, 2002.

28. O. Aran and L. Akarun, "A multi-class classification strategy for Fisher scores: Application to signer independent sign language recognition," Pattern Recognit., vol. 43, no. 5, pp. 1776-1788, 2010.

29. S. Singh and S. Silakari, "Generalized Discriminant Analysis algorithm for feature reduction in Cyber," Int. J. Comput. Sci. Inf. Secur., vol. 6, no. 1, pp. 173-180, 2009.

30. B. M. Asl, S. K. Setarehdan, and M. Mohebbi, "Support vector machine-based arrhythmia classification using reduced features of heart rate variability signal," Artif. Intell. Med., vol. 44, no. 1, pp. 5164, 2008.

31. S. Dong et al., "Bearing degradation state recognition based on kernel PCA and wavelet kernel SVM," Proc. Inst. Mech. Eng. Part C J. Mech. Eng. Sci., vol. 229, no. 15, pp. 2827-2834, 2015

32. K. R. Müller, S. Mika, G. Rätsch, K. Tsuda, and B. Schölkopf, "An introduction to kernel-based learning algorithms," IEEE Transactions on Neural Networks, vol. 12, no. 2. pp. 181-201, 2001.

33. V. N. Vapnik, "The Nature of Statistical Learning Theory," Springer, vol. 8. p. 188, 1995.

34. O. P. Yadav, D. Joshi, and G. L. Pahuja, "Support Vector Machine based Bearing Fault Detection of Induction Motor," Indian J. Adv. Electron. Eng., vol. 1, no. 1, pp. 34-39, 2013.

35. Y.-H. Liu, Y.-T. Chen, and S.-S. Lu, "Face Detection Using Kernel PCA and Imbalanced SVM," 2006, pp. 351-360.

36. M. GhasemiGol, R. Monsefi, and H. S. Yazdi, "Ellipse support vector data description," Commun. Comput. Inf. Sci., vol. 43 CCIS, pp. 257 $268,2009$.

37. A. Tabrizi, L. Garibaldi, A. Fasana, and S. Marchesiello, "Early damage detection of roller bearings using wavelet packet decomposition, ensemble empirical mode decomposition and support vector machine," Meccanica, vol. 50, no. 3, pp. 865-874, 2015.

38. L. Y. Zhao, L. Wang, and R. Q. Yan, "Rolling bearing fault diagnosis based on wavelet packet decomposition and multi-scale permutation entropy," Entropy, vol. 17, no. 9, pp. 6447-6461, 2015.

39. S. De Wu, P. H. Wu, C. W. Wu, J. J. Ding, and C. C. Wang, "Bearing fault diagnosis based on multiscale permutation entropy and support vector machine," Entropy, vol. 14, no. 8, pp. 1343-1356, 2012.

40. V. T. Tran, F. AlThobiani, A. Ball, and B. K. Choi, "An application to transient current signal based induction motor fault diagnosis of Fourier-Bessel expansion and simplified fuzzy ARTMAP," Expert Syst. Appl., vol. 40, no. 13, pp. 5372-5384, 2013.

41. J. Altmann and J. Mathew, "Multiple band-pass autoregressive demodulation for rolling-element bearing fault diagnosis," Mech Syst. Signal Process., vol. 15, no. 5, pp. 963-977, 2001

42. D. T. Hoang and H. J. Kang, "Rolling element bearing fault diagnosis using convolutional neural network and vibration image," Cogn. Syst. Res., vol. 53, no. 5, pp. 42-50, 2019.

43. X. Li, W. Zhang, and Q. Ding, "Cross-domain fault diagnosis of rolling element bearings using deep generative neural networks," IEEE Trans. Ind. Electron., vol. 66, no. 7, pp. 5525-5534, 2019.

44. K. Rama Krishna and K. I. Ramachandran, "Machinery Bearing Fault Diagnosis Using Variational Mode Decomposition and Support Vector Machine as a Classifier," in IOP Conference Series: Materials Science and Engineering, 2018, vol. 310, no. 1, pp. 10-20.

45. J. Xiong, Q. Zhang, Q. Liang, and H. Zhu, "Combining the MultiGenetic Algorithm and Support Vector Machine for Fault Diagnosis of Bearings," Shock Vib., vol. 1, no. 1, p. 3091618, 2018.

46. J. Sun, C. Yan, and J. Wen, "Intelligent bearing fault diagnosis method combining compressed data acquisition and deep learning," IEEE Trans. Instrum. Meas., vol. 67, no. 1, pp. 185-195, 2018.

47. M. M. Tahir, A. Q. Khan, N. Iqbal, A. Hussain, and S. Badshah, "Enhancing Fault Classification Accuracy of Ball Bearing Using Central Tendency Based Time Domain Features," IEEE Access, vol. 5, no. 1 , pp. 72-83, 2017. 


\section{Author Profile}

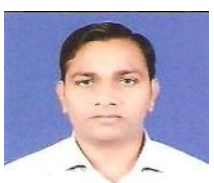

Rajeev Kumar Chauhan (M'15) received the B.E degree in Electrical Engineering from Madan Mohan Malviya University of Technology (Formerly M.M.M. Engineering College), Gorakhpur, Uttar Pradesh, India and the M.Tech. degree in Electrical Engineering (Power System) from Uttar Pradesh Technical University, Lucknow, India. He is currently pursuing the Ph.D degree at Dr. A. P. J. Abdul Kalam Technical University (Formerly Uttar Pradesh Technical University), Lucknow, India. Presently, he is working as an Associate Professor with the Department of Electrical \& Electronics Engineering, IMS Engineering College, Ghaziabad, India. His research interests include power system, power quality, power electronics and DSP application in power system. He is a member of IEEE and ISTE. He has published research papers in SCI and Scopus indexed journals

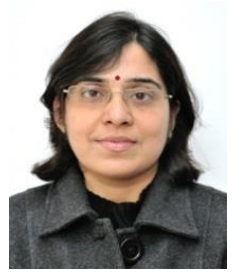

Dipti Saxena (SM'14) received the B.Tech. degree in Electrical Engineering from Kamla Nehru Institute of Technology, Sultanpur, Uttar Pradesh, India, the M.Tech. degree in Electrical Engineering (Process Control)) from Netaji Subash Institute of Technology, New Delhi, India, and the Ph.D degree from Gautam Buddha Technical University (Presently Dr. A. P. J. Abdul Kalam Technical University), Lucknow, India. Presently, she is working as an Associate Professor with the Department of Electrical Engineering, MNIT, Jaipur, India. Her research interests include power systems, power quality, power electronics and DSP application in power system. She has published research papers in IEEE transaction, SCI and scopus indexed journals. She is a senior member of IEEE.

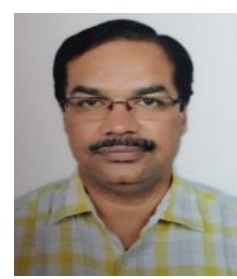

Jai Prakash Pandey received the B.Tech and M.Tech. degree in Electrical Engineering from Kamala Nehru Institute of Technology, Sultanpur, Uttar Pradesh, India. He obtained his Ph.D degree from Uttar Pradesh Technical University (Presently Dr. A. P. J. Abdul Kalam Technical University), Lucknow, Uttar Pradesh, India. Presently he is working as a Professor with the Department of Electrical Engineering, Kamala Nehru Institute of Technology, Sultanpur, Uttar Pradesh, India. His research interests include applications of artificial intelligence techniques to electrical engineering problems in power system, estate estimation and power quality. He has worked as Controller of examination at AKTU, Lucknow and MTU, Noida. Presently he is working as a Director at KNIT Sultanpur. 